\title{
Worm algorithms for the 3-state Potts model with magnetic field and chemical potential
}

\author{
Ydalia Delgado Mercado ${ }^{a}$, Hans Gerd Evertz $^{b}$, Christof Gattringer $^{a}$ \\ ${ }^{a}$ Karl-Franzens University Graz \\ Institute for Physics \\ A-8010 Graz, Austria \\ ${ }^{b}$ Technical University Graz \\ Institute for Theoretical and Computational Physics \\ A-8010 Graz, Austria
}

\begin{abstract}
We discuss worm algorithms for the 3-state Potts model with external field and chemical potential. The complex phase problem of this system can be overcome by using a flux representation where the new degrees of freedom are dimer and monomer variables. Working with this representation we discuss two different generalizations of the conventional Prokof'ev-Svistunov algorithm suitable for Monte Carlo simulations of the model at arbitrary chemical potential and evaluate their performance.
\end{abstract}

ydalia.delgado-mercado@uni-graz.at

evertz@tugraz.at

christof .gattringer@uni-graz.at 


\section{Introductory remarks}

Over the last two decades lattice methods have made considerable progress in our understanding of non-perturbative QCD, and now give rise to reliable quantitative results. However, one of the areas where progress is painfully slow is the analysis of QCD at finite density. The introduction of a chemical potential turns the fermion determinant into a complex number, such that it cannot be used as a weight factor in a Monte Carlo analysis - an obstacle known as complex phase problem. For true progress with QCD thermodynamics on the lattice new algorithmic concepts will be necessary. Often such new ideas are first developed and tested in simpler models before becoming applicable to the full theory.

The worm algorithm [1] is a conceptually new approach which has already been shown to be useful in some QCD related applications, in particular in strongly coupled lattice QCD [2, 3] and in scalar [4] or low dimensional lattice field theories [5. In this paper we extend the range of applications of worm algorithms to QCD related problems, and show that a worm algorithm can be used to simulate the 3-state Potts model in three dimensions with a center symmetry breaking term and a chemical potential, which in its standard representation has a complex phase problem. This model has been discussed as an effective theory for parts of the QCD phase diagram [6, 7, 8]. Numerical studies with various techniques for vanishing [9, 10] and non-vanishing chemical potential [8, 11, 12, 13] may be found in the literature. Recently a systematical analysis of the phase diagram as function of temperature and chemical potential was presented and its implications for QCD were discussed [14]. In this work we discuss in detail the worm algorithms used in [14] and assess their performance.

The 3 -state Potts model with magnetic field $\kappa$ and chemical potential $\mu$ in $d$ dimensions is described by the Hamiltonian

$$
H[P]=-\sum_{x}\left(\tau \sum_{\nu=1}^{d}\left[P(x) P(x+\hat{\nu})^{*}+\text { c.c. }\right]+\eta P(x)+\bar{\eta} P(x)^{*}\right),
$$

where we use the variables $\eta$ and $\bar{\eta}$ which are related to the strength $\kappa$ of the external field and the chemical potential $\mu$ via

$$
\eta=\kappa e^{\mu} \quad, \quad \bar{\eta}=\kappa e^{-\mu}
$$

The first sum in (10) is over all sites $x$ of a $d$-dimensional (hyper-) cubic lattice with periodic boundary conditions and a total of $V$ lattice points. The second sum is over all directions $\nu=1,2, \ldots d$, where $\hat{\nu}$ denotes the 
unit vector in direction $\nu$. The spin variables $P(x)$ are complex phases with three different values, $P(x) \in \mathbb{Z}_{3}=\left\{1, e^{i 2 \pi / 3}, e^{-i 2 \pi / 3}\right\}$. The coupling parameters $\tau, \kappa$ and $\mu$ are real and non-negative. The partition function of the model is given by $Z=\sum_{\{P\}} e^{-S[P]}$, where the sum runs over all possible configurations of the phases $P(x) \in \mathbb{Z}_{3}$.

In the context of an effective theory for QCD thermodynamics one considers the $d=3$ model and the spin variables $P(x)$ are interpreted as static quark sources at a spatial position $x$ (local Polyakov loops). A vanishing expectation value $P=V^{-1}\left\langle\sum_{x} P(x)\right\rangle$, which we here will often refer to as the magnetization $P$, signals confinement of quarks, while $P \neq 0$ corresponds to deconfined quarks. The parameter $\tau$ is a monotonically increasing function of the QCD temperature. $\kappa$ is proportional to the number of flavors and decreases with increasing quark mass. The value $\kappa=0$ then corresponds to pure gluodynamics. $\mu$ has the interpretation of the quark chemical potential in units of the inverse temperature.

For vanishing external field, $\kappa=0$ (i.e., $\eta=\bar{\eta}=0$ ), the 3 -state Potts model in three dimensions is known to have a first order transition at $\tau=0.183522(3)$ [10, 15]. For small external field and vanishing chemical potential (i.e., $\eta=\bar{\eta}=\kappa$ ) the first order transition persists, giving rise to a short first order line which ends in a critical endpoint at $(\tau, \kappa)=(0.183127(7), 0.00026(3))$ [10]. The case of non-vanishing chemical potential has been analyzed with techniques based on the Swendsen-Wang cluster algorithm using improved estimator techniques in [8] and reweighting in [12]. Within the flux representation [7] also local Metropolis updates were used [8, 11]. It has been demonstrated that turning on the chemical potential mildens the transition and shifts the critical endpoint towards smaller values of $\kappa$.

In this article we show that in the flux representation [7] the model is accessible also with suitably constructed worm algorithms. We discuss two different types of generalized worm algorithms: Type-I: A simple generalization of the conventional Prokof'ev-Svistunov worm [1] with steps where monomers are inserted, combined with a random hop to a lattice site with another monomer insertion. Type-II: Here the worms are open strings with monomers at their endpoints (contrary to the Type-I worms which are closed but with the possibility of intermediate hops).

The two algorithms are compared and evaluated using bulk observables: the magnetization $P$, the internal energy $U$, the susceptibility $\chi_{P}$ and the heat capacity $C$. 


\section{Flux representation}

In this section we briefly review the flux representation [7] that we use for the two worm algorithms to set the notation and to discuss the flux representation of the observables. For the nearest neighbor terms of (1) we use the ansatz

$$
e^{\tau\left[P(x) P(x+\hat{\nu})^{*}+c . c .\right]}=C \sum_{b_{x, \nu}=-1}^{+1} B^{\left|b_{x, \nu}\right|}\left(P(x) P(x+\hat{\nu})^{*}\right)^{b_{x, \nu}} .
$$

In this expression the term living on the link $(x, \nu)$ is written as a sum over

a dimer variable $b_{x, \nu} \in\{-1,0,+1\}$. A straightforward calculation gives the constants $C$ and $B$,

$$
C=\frac{e^{2 \tau}+2 e^{-\tau}}{3} \quad, \quad B=\frac{e^{2 \tau}-e^{-\tau}}{e^{2 \tau}+2 e^{-\tau}} .
$$

For the magnetic field terms, which live on a single site $x$, we use a similar ansatz,

$$
e^{\eta P(x)+\bar{\eta} P(x)^{*}}=\sum_{s_{x}=-1}^{+1} M_{s_{x}} P(x)^{s_{x}},
$$

which expresses the lhs. as a sum over a monomer variable $s_{x} \in\{-1,0,+1\}$ attached to the site $x$. Again one easily works out the monomer weights $M_{s}$ for $s=-1,0,+1$ and obtains

$$
M_{s}=\frac{1}{3}\left[e^{\eta+\bar{\eta}}+2 e^{-(\eta+\bar{\eta}) / 2} \cos \left((\eta-\bar{\eta}) \frac{\sqrt{3}}{2}-s \frac{2 \pi}{3}\right)\right] .
$$

The weights $M_{s}$ turn out to be non-negative for all values of $\eta$ and $\bar{\eta}$.

With the expressions (3) and (5) the partition sum assumes the form ( $V$ again denotes the total number of lattice points)

$$
Z=C^{d V} \sum_{\{b\}} \sum_{\{s\}} W[b, s]\left(\prod_{x} \sum_{P(x)} P(x)^{\sum_{\nu}\left[b_{x, \nu}-b_{x-\hat{\nu}, \nu}\right]+s_{x}}\right) .
$$

The first two sums in (7) run over all configurations of the dimer variables $b_{x, \mu}$ and of the monomer variables $s_{x}$. We have introduced the weight factor

$$
W[b, s]=\left(\prod_{x, \nu} B^{\left|b_{x, \nu}\right|}\right)\left(\prod_{x} M_{s_{x}}\right) .
$$


The summation over the phases $P(x)$ can now be performed in closed form. Using that the sum over the roots of unity vanishes, one finds ( $n$ is an integer),

$$
\sum_{P} P^{n}=3 T(n) \quad \text { with } \quad T(n)=\delta_{n \bmod 3,0},
$$

where we introduced the triality function $T(n)$ which equals 1 if $n$ is a multiple of 3 and vanishes otherwise. The partition sum in its final form reads

$$
Z=\left(3 C^{d}\right)^{V} \sum_{\{b\}} \sum_{\{s\}} W[b, s] \prod_{x} T\left(\sum_{\nu}\left[b_{x, \nu}-b_{x-\hat{\nu}, \nu}\right]+s_{x}\right) .
$$

In (10) the Potts model is represented in terms of the dimer variables $b_{x, \nu} \in$ $\{-1,0,+1\}$ which live on the links $(x, \nu)$ of the lattice, and the monomer variables $s_{x} \in\{-1,0,+1\}$ attached to the sites $x$. Each configuration of dimers and monomers comes with a real and non-negative weight $W[b, s]$ as given in (8) and thus the complex phase problem is solved. The weight consists of a factor $B$ for every non-vanishing dimer, and a monomer factor $M_{s_{x}}$ for each site $x$, according to the monomer variable $s_{x}$ at that site.

The configurations of dimers and monomers must obey the constraint given by the second factor in (10). This factor forces the total flux of dimers and monomers at each site $x$ to be a multiple of 3 .

In Fig. 1 we show (for the 2-dimensional case) a few admissible flux configurations at individual sites: dimers are represented by arrows in the horizontal plane and monomers by arrows on the vertical axis. If an arrow points towards a site (away from the site) it contributes with $+1(-1)$ to the total flux at that site. Trivial dimers, $b_{x, \nu}=0$, and trivial monomers, $s_{x}=0$, do not contribute to the constraint and thus need not be represented in the illustration of admissible fluxes.
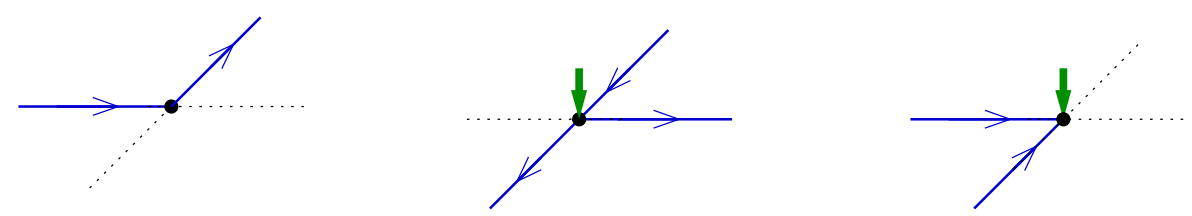

Figure 1: Examples of admissible fluxes at a site $x$ for the case of $d=2$ dimensions. See the text for the description of the representation.

In our tests of the worm algorithms we use the bulk observables: internal energy $U$, the magnetization $P$, i.e., the expectation value of the averaged 
spin variable $P=V^{-1} \sum_{x}\langle P(x)\rangle$, the heat capacity $C$ and the susceptibility $\chi_{P}$. These quantities may be obtained as suitable derivatives of $\ln Z$. For the evaluation of these derivatives it is useful to note the following relations for derivatives of the monomer weights $M_{s}$ :

$$
\frac{\partial M_{+1}}{\partial \eta}=\frac{\partial M_{-1}}{\partial \bar{\eta}}=M_{0}, \frac{\partial M_{0}}{\partial \eta}=\frac{\partial M_{+1}}{\partial \bar{\eta}}=M_{-1}, \frac{\partial M_{-1}}{\partial \eta}=\frac{\partial M_{0}}{\partial \bar{\eta}}=M_{+1} .
$$

Exploiting these relations one obtains for the expectation value of the spatially averaged spin the following representation in terms of monomers

$$
P \equiv \frac{1}{V} \frac{\partial}{\partial \eta} \ln Z=\frac{1}{V}\left\langle\mathcal{S}_{+1} \frac{M_{0}}{M_{+1}}+\mathcal{S}_{0} \frac{M_{-1}}{M_{0}}+\mathcal{S}_{-1} \frac{M_{+1}}{M_{-1}}\right\rangle
$$

where $\mathcal{S}_{+1}$ denotes the number of sites $x$ where the monomer variables have the value $s_{x}=+1$, etcetera.

In a similar way one obtains for the internal energy

$$
\begin{aligned}
U & \equiv\langle H\rangle=\left[-\tau \frac{\partial}{\partial \tau}-\eta \frac{\partial}{\partial \eta}--\bar{\eta} \frac{\partial}{\partial \bar{\eta}}\right] \ln Z \\
& =-2 d V \tau B-\left\langle\mathcal{B} \widetilde{B}+\mathcal{S}_{+1} \widetilde{M}_{+1}+\mathcal{S}_{0} \widetilde{M}_{0}+\mathcal{S}_{-1} \widetilde{M}_{-1}\right\rangle
\end{aligned}
$$

with $\mathcal{B}$ denoting the number of links $(x, \nu)$ where the dimer variables are non-zero, $b_{x, \nu} \neq 0$. The new numerical constants in this expression are

$$
\begin{array}{rlrl}
\widetilde{B} & =\frac{9 \tau}{e^{3 \tau}+1-e^{-3 \tau}}, & \widetilde{M}_{+1} & =\frac{\eta M_{0}+\bar{\eta} M_{-1}}{M_{+1}}, \\
\widetilde{M}_{0} & =\frac{\eta M_{-1}+\bar{\eta} M_{+1}}{M_{0}} \quad, \quad \widetilde{M}_{-1}=\frac{\eta M_{+1}+\bar{\eta} M_{0}}{M_{-1}} .
\end{array}
$$

Using equivalent steps also the heat capacity $C$ and the susceptibility $\chi_{P}$ can be expressed in terms of the monomer and dimer numbers and their fluctuations. For the evaluation of spin correlators one may use locally varying weights $\eta$ and $\bar{\eta}$ as generating functionals. The spin correlators are then related to correlators of monomers. 


\section{Worm algorithms}

In a worm algorithm [1, the constraint of flux conservation (9) is temporarily relaxed during a Monte Carlo move. One unit of flux is inserted at some lattice site and then moved through the lattice by local steps, until the insertion is healed again, either by coming back to the original site, or by a change of the local monomer number. The individual local steps are performed with Metropolis update probabilities. Each complete worm makes up one update step in the model with constraint (9) which is to be simulated.

As outlined in the introduction, we use two different types of worm algorithms. Type-I: The worms are closed, and in addition to the usual local worm propagation, there are random hops with insertion of monomers. Type-II: The worms are open strings on the lattice with monomers at the endpoints.

\subsection{Type-I: Closed worm algorithm}

Each worm is generated using four different moves. We illustrate them in Fig. 2 for the simplest case of an initially empty lattice, i.e., all dimerand monomer variables are set to 0 in the beginning. The worm starts at a random position of the lattice (1). It may decide to insert dimers and move to the neighboring site (2) but also monomers can be inserted (3). The insertion of a monomer is followed by a sequence of attempts to insert another monomer at a randomly chosen site, which will succeed (3) with a probability depending on the weights $M_{s}$. These steps are continued until the worm closes (4). The acceptance of each step is governed by a Metropolis decision.

The subsequent pseudo-code describes the algorithm for the general case of $d$ dimensions (for the numerical tests discussed later we set $d=3$ ). By $x_{0}$

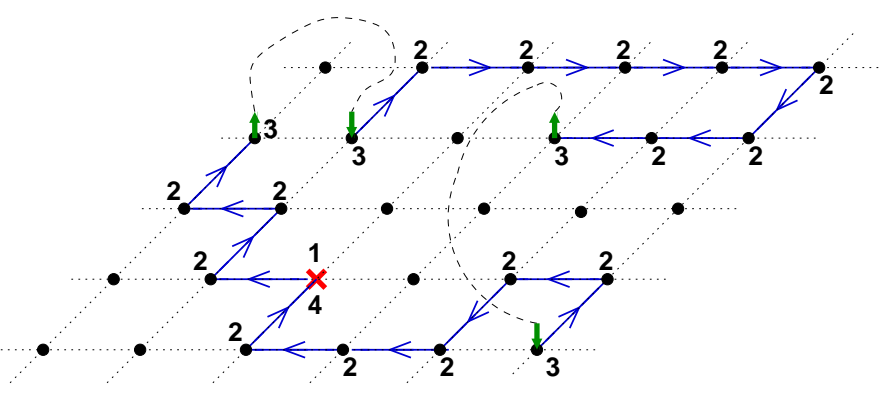

Figure 2: Example of a Type-I closed worm on an initially empty 2-D lattice. 
we denote the starting point of the worm and $x$ is the current position of its head. worm_sign is a variable that determines whether the worm raises or lowers the flux. insert_monomer_flag is a logical variable controlling the status of the worm. By $x \oplus y$ we denote addition modulo 3 which is the usual addition operation except in the cases $x=y=1$ and $x=y=-1$ where we define $+1 \oplus+1=-1$ and $-1 \oplus-1=+1$. Finally $\operatorname{rand}()$ is a random number generator for uniformly distributed real numbers in the interval $[0,1)$.

\section{Pseudocode for Type-I closed worms:}

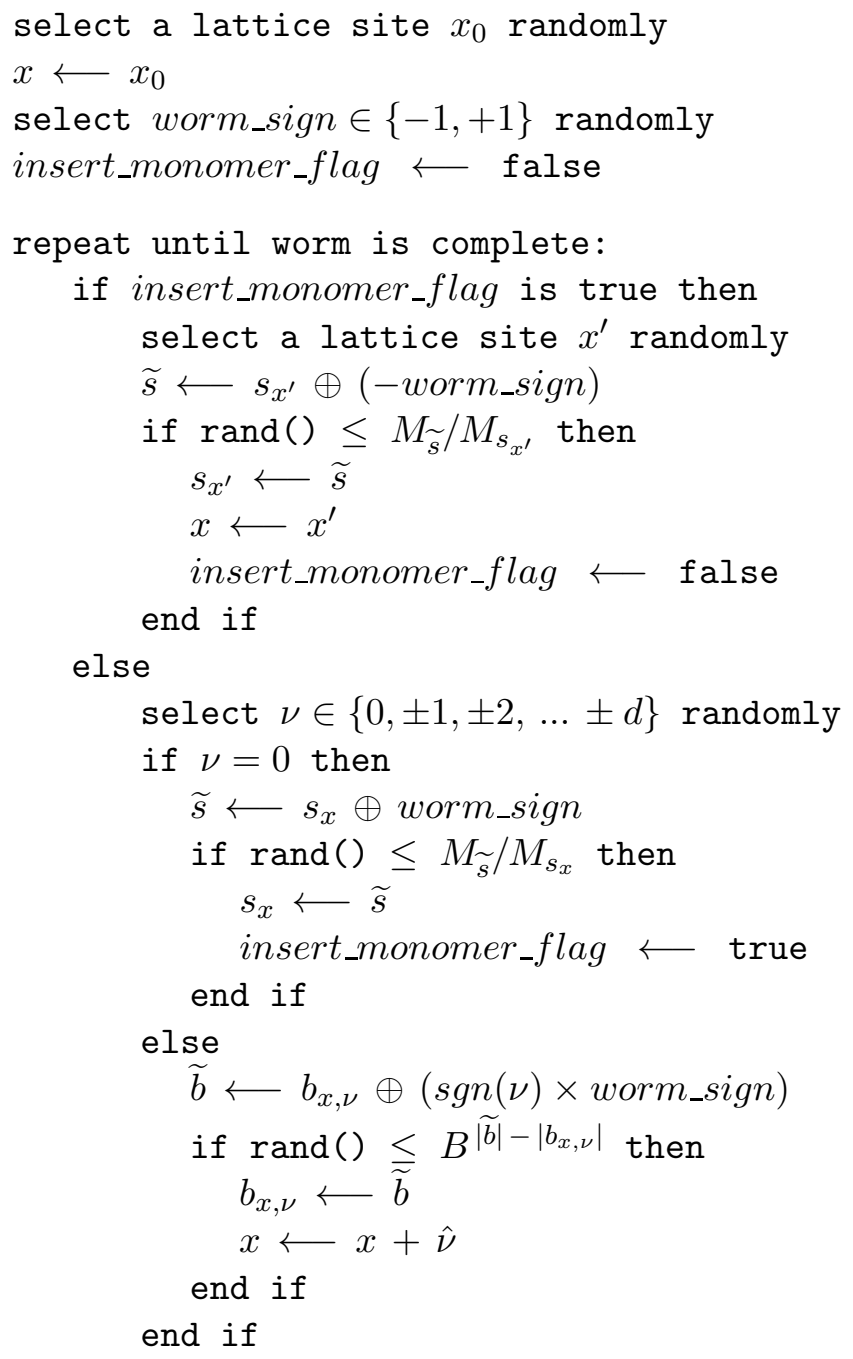




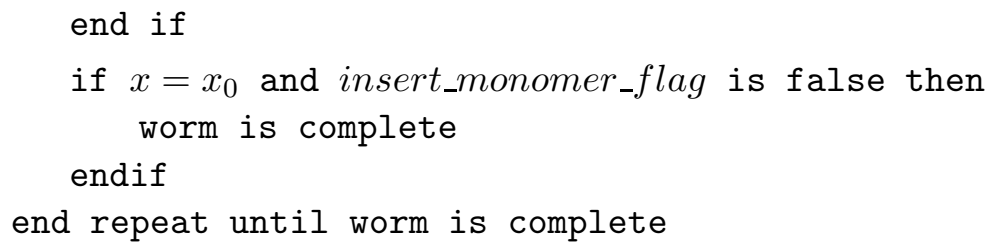

It is straightforward to show detailed balance using the Boltzmann weight $W[b, s]$ given in (8), and that the algorithm is ergodic.

\subsection{Type-II: Open worm algorithm}

We also employed a second variant of the algorithm which we refer to as Type-II open worms. In this case, each worm is generated using three moves which we again illustrate for an initially empty lattice (Fig. 3): The worm tries to start at a random position of the lattice by inserting a monomer (1). It then may decide to insert dimers or monomers. If a dimer is inserted the worm moves to the neighboring site (2). If a monomer is inserted, the worm is complete and terminates (3). Again the acceptance of the moves is decided with a Metropolis step.

We stress that the Type-II open worm will not work in the case of vanishing external magnetic field, i.e., for $\kappa=0$. In that case no monomers appear and the Type-II worm cannot start. For small $\kappa$ the Type-II open worm will have a very small probability for starting, since the monomer weights are linear in $\kappa$ (for small values of $\kappa$ ). For larger values of $\kappa$ there is no starting problem of the Type-II worms (see also Section 4.2). For $\kappa \neq 0$ it is straightforward to show detailed balance and ergodicity for Type-II worms, and we found that they are even simpler to implement than the closed worms.

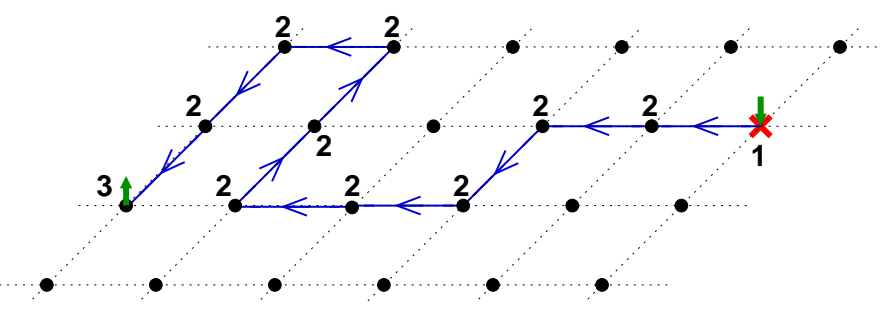

Figure 3: Example of a Type-II open worm on an initially empty 2dimensional lattice. See the text for its discussion. 


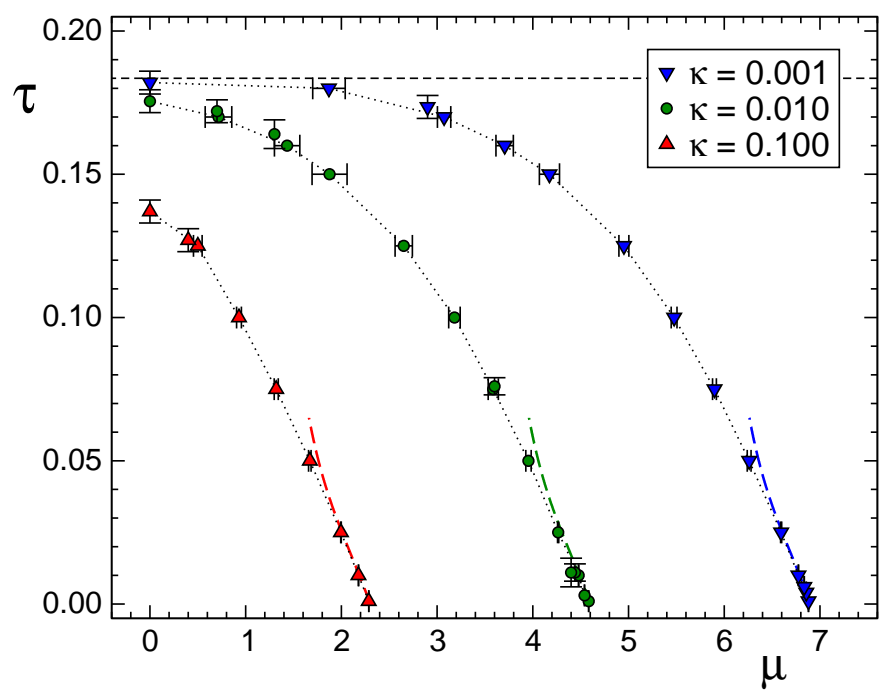

Figure 4: Phase diagram [14] as obtained from the maxima of the susceptibility of $P$. We show results for three values of $\kappa$. The dashed curves at the bottom are the results of a perturbative expansion in $\tau$ and the horizontal line marks the critical value of $\tau$ for the $\kappa=0$ case.

\section{Numerical assessment of the worms}

In this section we show the results of our tests and performance analysis for the two generalized worm algorithms. For a detailed discussion of the physics results obtained in the simulations of the generalized Potts model we refer the reader to [14. Here we only include a figure of the phase diagram to allow the reader to locate the parameter values of the simulations discussed in this section in the phase diagram. In Fig. 4 we show the phase diagram in the $\tau-\mu$ plane obtained from the position of the maxima of $\chi_{P}$ for three values of $\kappa$. The dashed curves at the bottom are the results of a perturbative expansion in $\tau$ and the horizontal line marks the critical value of $\tau$ for the $\kappa=0$ case. To the left of the phase lines the Polyakov loop is small (confinement), while it is close to 1 to the right of the phase lines (deconfined phase). The transitions are of a crossover nature.

\subsection{Validity of the algorithms and the flux-based observables}

As a first test we compare the results from the two worm algorithms with the results from an exact evaluation on a small $2 \times 2 \times 3$ lattice using the original spin formulation of the model. We consider the observables $U, C, P$ and $\chi_{P}$ 


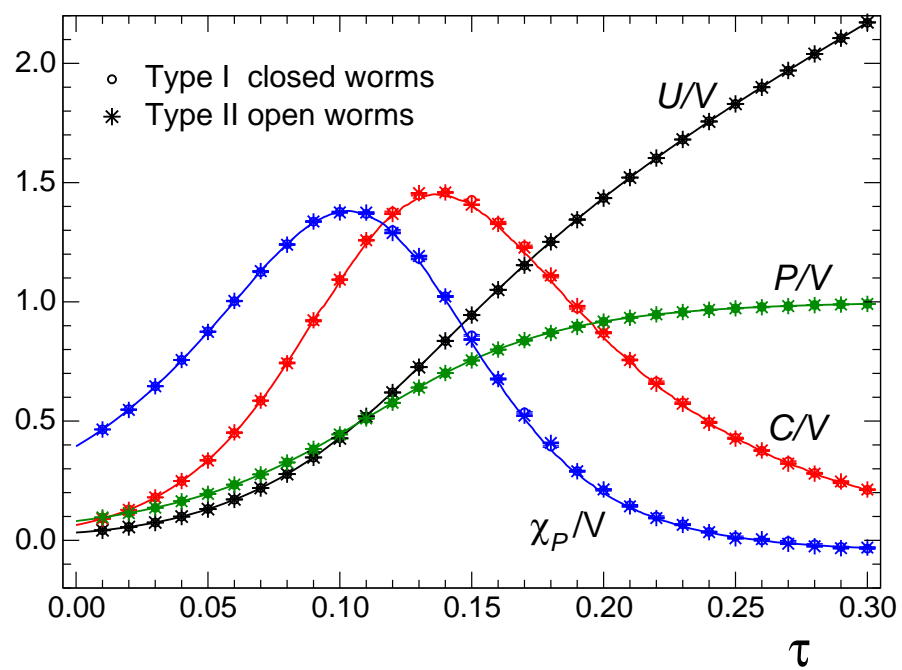

Figure 5: Comparison of the exact result (full curves) on a $2 \times 2 \times 3$ lattice with the two worm algorithms (symbols). We show the results for $U, C, P$ and $\chi_{P}$ as a function of $\tau$ using $\kappa=0.001$ and $\mu=6.0$. Note that the data points from the two algorithms agree almost perfectly such that the corresponding symbols essentially fall on top of each other.

normalized by the volume. In Fig. [5] we show these observables as a function of $\tau$ for $\kappa=0.001$ and $\mu=6.0$. The curves represent the results from the exact evaluation, while for the data from the worm algorithms symbols are used. It is obvious, that the numbers from the exact evaluation and from the worm algorithms agree very well. This not only establishes the validity of the worm algorithms, but also of the mapping of the physical observables onto the flux representation. The test on the small volume was repeated for different values of the parameters $\tau, \kappa$ and $\mu$ and we always found excellent agreement of the worm results with the exact evaluation of the observables.

In addition to the comparison to exact results on small volumes we performed also a comparison with a conventional Metropolis calculation in the spin representation on larger volumes. This comparison is of course limited to vanishing chemical potential, $\mu=0$, where the complex phase problem is absent also in the spin representation and the conventional Metropolis approach is possible. In Fig. 6 we compare the results for the internal energy $U$ and the heat capacity $C$ as a function of $\tau$ calculated with the three algorithms: Conventional Metropolis (red circles), closed worm (blue upward pointing triangles) and open worm (green downward pointing triangles) for $\kappa=0.01$ and $\mu=0$ on a $16^{3}$ lattice for values of $\tau$ near the crossover. The 

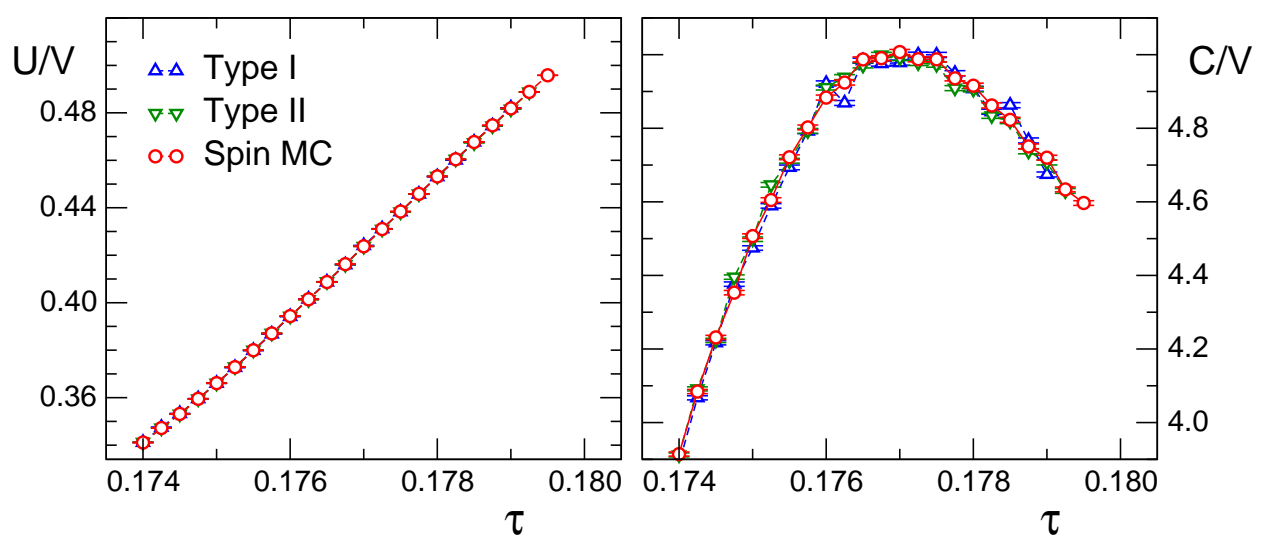

Figure 6: Internal energy $U$ (left) and heat capacity $C$ (right) for $\kappa=$ 0.01 and $\mu=0$ on a $16^{3}$ lattice at values of $\tau$ near the crossover. We compare the results from the conventional Metropolis simulation in the spin representation (red circles), the Type-I closed worm algorithm (blue upward pointing triangles) and the Type-II open worms (green downward pointing triangles).

Metropolis simulation uses $10^{6}$ sweeps of local Metropolis steps for equilibration and $10^{6}$ measurements separated by 20 sweeps for decorrelation. For both worm algorithms we used $10^{6}$ worms for equilibration and $10^{6}$ measurements. For the Type-I closed worm we separated two measurements by $N_{\text {deco }}=20$ worms for decorrelation, while for the Type-II open worms 5000 worms were used for decorrelation. The results from the three algorithms fall on top of each other. The same tests were conducted at other values of the parameters and also for $P$ and $\chi_{P}$. Furthermore, for $\mu>0$ where simulations with the conventional spin-based Metropolis algorithm are not possible, we performed a comparison among the two worm algorithms and also in this case found excellent agreement. We conclude that the two worm algorithms are valid, and that the representation of the observables in terms of fluxes was implemented correctly.

\subsection{Time series}

In this subsection we now study performance aspects of the two worm algorithms. We begin our assessment with analyzing the time series of measurements of the total number $\mathcal{B}$ of non-zero dimer variables and the number $\mathcal{S}$ of non-zero monomers. As shown at the end of Section 2, the physical 

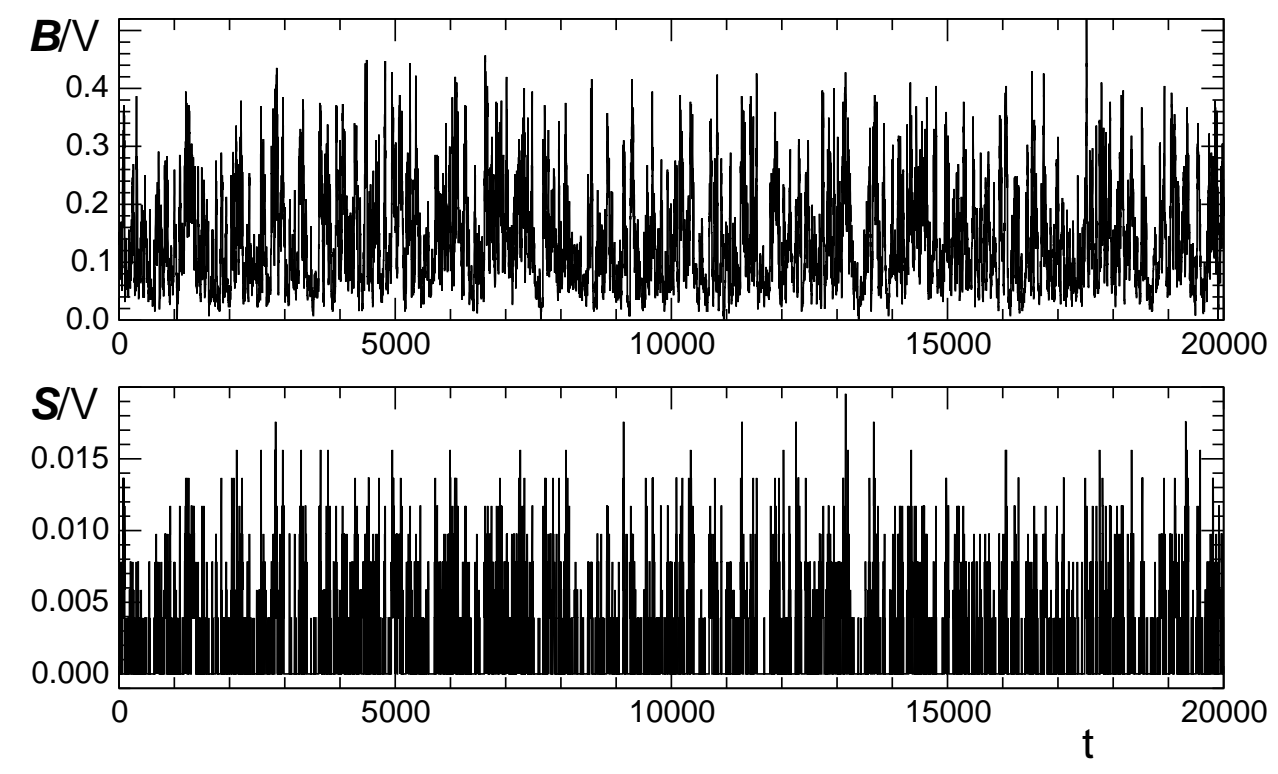

Figure 7: Time series for $\mathcal{B} / V$ and $\mathcal{S} / V$ at $\tau=0.1782, \kappa=0.005$, and $\mu=0$ on a $8^{3}$ lattice, generated with Type-I worms. The Monte Carlo time on the horizontal axis is measured in units of $N_{\text {deco }}=20$ worms.

observables can be constructed from $\mathcal{B}$ and $\mathcal{S}$ and their moments. In Fig. 7 we show time series from Type-I worms for $\mathcal{B} / V$ in the top plot and for $\mathcal{S} / V$ (bottom) for the parameters $\tau=0.1782, \kappa=0.005, \mu=0.0$ and a volume of $8^{3}$ (time series for Type-II worms behave similarly). Between two measurements we used $N_{\text {deco }}=20$ worms for decorrelation, so the Metropolis time on the horizontal axis is measured in units of 20 worms. We do not observe structures on scales larger than several hundred worms and as a first impression conclude that the Type-I worms lead to reasonably quick sampling. The sampling becomes better for larger values of $\kappa$ or $\mu$.

There are, however, regions in the space of couplings where one finds that the flux representation and worms are not the optimal approach. In general this is the case for very small values of $\kappa$ (typically for $\kappa$ of $O\left(10^{-4}\right)$ ), where the monomer weights are small (in leading order they are linear in $\kappa$ ), and thus it is hard to generate monomers. This is illustrated in Fig. 8, where we show the same time series as in Fig. 7 , but now at $\kappa=0.0002, \mu=0$ and $\tau=0.183$, again with $N_{\text {deco }}=20$ worms for decorrelation. Although the time series for $\mathcal{B} / V$ still looks reasonable, the time series for $\mathcal{S} / V$ shows that monomers are generated only very rarely and very high statistics would 

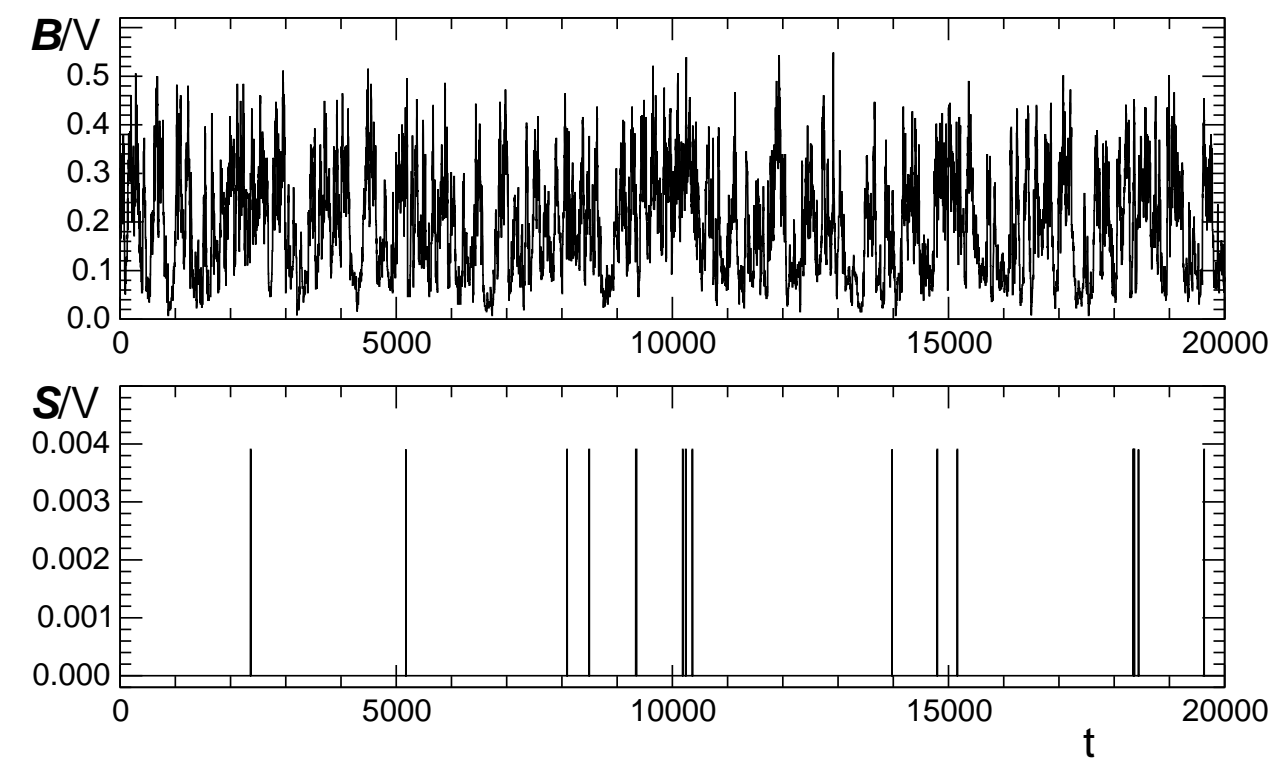

Figure 8: Time series for $\mathcal{B} / V$ and $\mathcal{S} / V$ at $\kappa=0.0002, \mu=0$ and $\tau=0.183$ on a $8^{3}$ lattice, generated with Type-I worms. The Monte Carlo time on the horizontal axis is measured in units of $N_{\text {deco }}=20$ worms.

be needed to obtain reliable results. We stress that this is a property of the flux representation: As mentioned, the monomer weights are very small at small $\kappa$, so monomers are indeed expected to be very rare and the flux representation is not the optimal choice for very small $\kappa$. This difficulty could be overcome by reweighting the sector of phase space with small monomer numbers to obtain a higher weight and thus higher frequency in the Monte Carlo. They could then be weighted back in order to compute expectation values. In the present study we did not explore such a reweighting strategy. We also note that for small $\kappa$ the complex phase problem is very mild and reweighting techniques were used to successfully simulate the system with the conventional spin representation [8, 12] in that region of the parameter space also at small $\mu$.

\subsection{Characteristic quantities}

Let us now come to a quantitative comparison of the Type-I closed worms and Type-II open worms. For a sensible comparison one has to consider the actual changes done by the worms: A Type-II open worm inserts two 
monomers at its endpoints and connects it with dimers (one segment), while a Type-I closed worm may consist of several segments of dimer strings connected by large hops between monomers (it may also consist of only dimer hops without large hops, i.e., only one closed segment). We first look at some characteristic quantities in order to describe the behavior of the worms in different regions of parameter space. By $n_{\text {os }}$ we denote the average number of open segments in Type-I worms, i.e., segments with monomer hops at their endpoints (if a Type-I worm closes without monomer insertions $n_{o s}$ is zero). By $D$ we denote the average number of dimer steps in a Type-I or Type-II worm. Another interesting quantity for the comparison of the two worms is the ratio $r$ of the number of successful worm starts divided by the number of all start attempts. Depending on the parameters, here one expects a drastic difference between the worms, since the open worms can start only with the insertion of a monomer. Finally, for the comparison of the computational cost of the two algorithms we define the cost ratio $c_{s}$ for the Type-I and Type-II worms as the ratio of the total number of attempted steps (dimer, monomer and start attempts) to the number of accepted steps.

In Table 1 we collect the data for Type-I closed worms, and in Table 2 for Type-II worms. The analysis was done for five different sets of parameters (first column) and for four volumes (second column). Let us begin with the comparison of the ratio $r$ (number of successful worm starts divided by the number of all start attempts). For Type-I closed worms the starting probability is similar for all parameter sets (ranging between 0.129 and 0.209). Since here the chance for starting with a dimer insertion is $6 / 7$, the ratio goes down slightly when dimers are more costly, i.e., when $\tau$ is small, or when the system is monomer dominated ( $\kappa$ and/or $\mu$ large). For the Type-II open worms, $r$ shows a much larger variation: Here the worm must start with the insertion of a monomer, and for some parameter sets (Sets B, D and E) these are scarce, such that for the Type-II worms many start attempts fail. On the other hand, for parameters where monomers are abundant (Sets A and C), we observe a very high rate of successful starts.

For Type-I closed worms the abundance of monomers is also reflected in the average number $n_{\text {os }}$ of open segments: It is small for the same parameter sets (Sets B, D and E) where the starting ratio $r$ of the Type-II worms is small, i.e., for regions of parameter space with few monomers. Concerning the dependence on the volume $V$, we find that $n_{\text {os }}$ grows linearly with the volume, i.e., $n_{o s}=c V$ perfectly describes the data, where $c$ is a constant that depends on the parameters $\tau, \kappa$ and $\mu$.

The behavior of the average number $D$ of dimer steps is interesting. For Type-II open worms we find that $D$ varies only very little with the 


\begin{tabular}{lrclcr}
\hline Parameters & $V$ & $r$ & $n_{o s} / V$ & $D / V$ & $c_{s}$ \\
\hline Set A: & $6^{3}$ & 0.166 & 0.122 & 0.383 & 3.81 \\
$\tau=0.100$, & $8^{3}$ & 0.166 & 0.122 & 0.380 & 3.80 \\
$\kappa=0.001$, & $16^{3}$ & 0.166 & 0.122 & 0.378 & 3.80 \\
$\mu=5.9$ & $32^{3}$ & 0.166 & 0.122 & 0.377 & 3.80 \\
\hline Set B: & $6^{3}$ & 0.209 & $2.50 \mathrm{e}-4$ & 0.332 & 3.26 \\
$\tau=0.181$, & $8^{3}$ & 0.200 & $2.29 \mathrm{e}-4$ & 0.221 & 3.51 \\
$\kappa=0.001$, & $16^{3}$ & 0.190 & $1.82 \mathrm{e}-4$ & 0.066 & 4.81 \\
$\mu=0.8$ & $32^{3}$ & 0.188 & $1.44 \mathrm{e}-4$ & 0.031 & 6.32 \\
\hline Set C: & $6^{3}$ & 0.129 & 0.445 & 0.212 & 3.89 \\
$\tau=0.025$, & $8^{3}$ & 0.129 & 0.444 & 0.212 & 3.89 \\
$\kappa=0.005$, & $16^{3}$ & 0.129 & 0.444 & 0.211 & 3.88 \\
$\mu=5.2$ & $32^{3}$ & 0.129 & 0.444 & 0.212 & 3.89 \\
\hline Set D: & $6^{3}$ & 0.173 & $7.59 \mathrm{e}-4$ & 0.126 & 3.99 \\
$\tau=0.170$, & $8^{3}$ & 0.170 & $5.66 \mathrm{e}-4$ & 0.059 & 4.64 \\
$\kappa=0.005$, & $16^{3}$ & 0.169 & $4.18 \mathrm{e}-4$ & 0.017 & 7.25 \\
$\mu=0.2$ & $32^{3}$ & 0.169 & $4.03 \mathrm{e}-4$ & 0.012 & 8.73 \\
\hline Set E: & $6^{3}$ & 0.164 & 0.013 & 0.197 & 4.61 \\
$\tau=0.150$, & $8^{3}$ & 0.164 & 0.012 & 0.178 & 4.70 \\
$\kappa=0.010$, & $16^{3}$ & 0.164 & 0.012 & 0.166 & 4.77 \\
$\mu=2.0$ & $32^{3}$ & 0.164 & 0.012 & 0.164 & 4.78 \\
\hline
\end{tabular}

Table 1: Characteristic quantities for Type-I closed worms (see the text for their definitions). All the simulations use $10^{6}$ worms for equilibration and $10^{7}$ measurements separated by $N_{\text {deco }}=5$ worms for decorrelation. The error is usually smaller than the last digit we show.

volume and is correlated with the abundance of monomers: Since monomers are needed to terminate Type-II worms, parameter values where monomers are scarce (Sets B, D and E) will lead to longer worms, i.e., increase $D$. For Type-I closed worms we find that $D$ essentially grows linearly with the volume for Sets A, C and E. For the sets B and D (those with very low density of monomers) we find that $D$ grows slower than linear. This is correlated with a very small number $n_{o s}$ of open segments for these ensembles.

The cost ratio $c_{s}$ essentially reflects the behavior of $r, n_{o s}$ and $D$ : Whenever monomers are sufficiently abundant, the values of $c_{s}$ are independent of the volume and are roughly equal for Type-I and Type-II worms. Discrepancies are observed only for the sets where monomers are scarce (Sets 


\begin{tabular}{lrllr}
\hline Parameters & $V$ & $r$ & $D$ & $c_{s}$ \\
\hline Set A: & $6^{3}$ & 0.268 & 2.93 & 3.78 \\
$\tau=0.100$, & $8^{3}$ & 0.268 & 2.93 & 3.78 \\
$\kappa=0.001$, & $16^{3}$ & 0.268 & 2.93 & 3.78 \\
$\mu=5.9$ & $32^{3}$ & 0.268 & 2.93 & 3.78 \\
\hline Set B: & $6^{3}$ & 0.0014 & 108 & 9.44 \\
$\tau=0.181$, & $8^{3}$ & 0.0015 & 163 & 7.17 \\
$\kappa=0.001$, & $16^{3}$ & 0.0016 & 195 & 6.42 \\
$\mu=0.8$ & $32^{3}$ & 0.0015 & 179 & 6.77 \\
\hline Set C: & $6^{3}$ & 0.625 & 0.445 & 3.85 \\
$\tau=0.025$, & $8^{3}$ & 0.626 & 0.445 & 3.85 \\
$\kappa=0.005$, & $16^{3}$ & 0.625 & 0.445 & 3.85 \\
$\mu=5.2$ & $32^{3}$ & 0.626 & 0.445 & 3.85 \\
\hline Set D: & $6^{3}$ & 0.0059 & 30.1 & 8.57 \\
$\tau=0.170$, & $8^{3}$ & 0.0058 & 29.2 & 8.77 \\
$\kappa=0.005$, & $16^{3}$ & 0.0058 & 28.3 & 8.98 \\
$\mu=0.2$ & $32^{3}$ & 0.0058 & 28.3 & 8.98 \\
\hline Set E: & $6^{3}$ & 0.0482 & 12.5 & 4.70 \\
$\tau=0.150$, & $8^{3}$ & 0.0482 & 12.5 & 4.70 \\
$\kappa=0.010$, & $16^{3}$ & 0.0482 & 12.5 & 4.70 \\
$\mu=2.0$ & $32^{3}$ & 0.0482 & 12.5 & 4.70 \\
\hline
\end{tabular}

Table 2: Characteristic quantities for Type-II open worms (see the text for their definition). All the simulations use $10^{6}$ worms for equilibration and $10^{7}$ measurements separated by $N_{\text {deco }}=500$ worms for decorrelation. The error is usually smaller than the last digit we show.

B and D): In these cases the closed Type-I worms have the advantage that they can run without any monomer insertions at all, thus improving their cost ratio. This advantage is, however, lost when the volume is larger and longer dimer chains emerge. Then from time to time a monomer insertion takes place and also Type-I worms then spend quite some time with trying to insert another monomer necessary for continuation. Thus for larger volumes the cost ratios $c_{s}$ of Type-I and Type-II worms roughly agree also for Sets B and D. From the assessment of $r, n_{o s}, D$ and $c_{s}$ we conclude that for these characteristic quantities the two algorithms behave similarly, with the exception that the closed Type-I worms are more flexible since they work also for vanishing external magnetic fields. 


\subsection{Autocorrelation times}

The relevant figure of merit for an algorithm is computational effort for a fixed precision of results. We therefore analyze the integrated autocorrelation time $\tau_{\text {int }}^{X}$ of the bulk observables $X=U, C, P$ and $\chi_{p}$. Since the sizes of worms vary drastically between the different cases, we need to normalize the autocorrelation times. For this purpose we define one "sweep" as $\tau_{0}=3 V / D$ worms, i.e., the average number of worms needed to visit every link of the lattice as the (customary) unit for the integrated autocorrelation times $\tau_{\text {int }}^{X}$. Obviously one may express $\tau_{0}$ also in terms of measurements as $\tau_{0}=3 \mathrm{~V} /\left(D N_{\text {deco }}\right)$ measurements. In Tables 3 and 4 we give the autocorrelation times in units of $\tau_{0}$. In order to obtain a measure of computational effort, the results are multiplied by the cost ratio $c_{s}$, in other words we show $\bar{\tau}=c_{s} \tau_{\text {meas }} / \tau_{0}$, where $\tau_{\text {meas }}$ simply is the unnormalized autocorrelation time in units of measurements. The statistical errors of autocorrelation times were estimated by a jackknife procedure and are between about 5 and 10 percent depending on the parameter values. This is sufficient for the subsequent comparison of the two algorithms.

The autocorrelation times are almost independent of volume for most parameter sets, reflecting the crossover or noncritical nature of correlations. Even for set B, which is very close to the critical value of $\tau$ at $\kappa=0$, autocorrelation times of Type-I are small and increase only very moderately with volume. Self averaging within a large volume can lead to the decreasing times (at high scale) for the internal energy $U$ for set B and Type-II.

Comparing the autocorrelation times (Tables 3 and 4) shows that TypeII open worms outperform the Type-I closed worms (i.e., shorter autocorrelation times) for those sets where monomers are abundant (Sets A and C), while the Type-I worms perform better for Sets B, D, E, where monomers are scarce. The reason is that Type-II worms always start with the insertion of a monomer, while Type-I worms insert monomers only with a probability $1 / 7$. Thus Type-II worms may have a larger portion of monomer insertions compared to dimer steps, and thus do many monomer changes which is advantageous for Sets A and C. Vice versa, for the sets with few monomers, the Type-I worms can do many dimers steps without needing monomer insertions and thus perform better in this situation. We remark that some of the disadvantage of the Type-I worms for parameter sets where monomers are abundant could be ameliated by changing the probability for offering a monomer change from $1 / 7$ to some larger value. Remarkably, the relative performance of the two worm types is roughly proportional to $r / c_{s}$, i.e. to the acceptance rates for attempted steps. 


\begin{tabular}{lrrrrr}
\hline Parameters & $V$ & $\bar{\tau}_{\text {int }}^{U}$ & $\bar{\tau}_{\text {int }}^{C}$ & $\bar{\tau}_{\text {int }}^{P}$ & $\bar{\tau}_{\text {int }}^{X P}$ \\
\hline Set A: & $6^{3}$ & 31 & 17 & 26 & 15 \\
$\tau=0.100$, & $8^{3}$ & 31 & 17 & 27 & 14 \\
$\kappa=0.001$, & $16^{3}$ & 31 & 17 & 26 & 14 \\
$\mu=5.9$ & $32^{3}$ & 32 & 18 & 27 & 15 \\
\hline Set B: & $6^{3}$ & 83 & 30 & 19 & 15 \\
$\tau=0.181$, & $8^{3}$ & 99 & 35 & 23 & 18 \\
$\kappa=0.001$, & $16^{3}$ & 151 & 72 & 78 & 21 \\
$\mu=0.8$ & $32^{3}$ & 156 & 73 & 89 & 32 \\
\hline Set C: & $6^{3}$ & 7.8 & 5.0 & 6.7 & 4.9 \\
$\tau=0.025$, & $8^{3}$ & 7.9 & 5.1 & 7.5 & 4.8 \\
$\kappa=0.005$, & $16^{3}$ & 7.7 & 5.2 & 8.0 & 5.0 \\
$\mu=5.2$ & $32^{3}$ & 7.7 & 5.2 & 7.9 & 5.1 \\
\hline Set D: & $6^{3}$ & 27 & 16 & 13 & 6.9 \\
$\tau=0.170$, & $8^{3}$ & 25 & 13 & 13 & 6.1 \\
$\kappa=0.005$, & $16^{3}$ & 32 & 14 & 11 & 5.9 \\
$\mu=0.2$ & $32^{3}$ & 54 & 28 & 11 & 6.2 \\
\hline Set E: & $6^{3}$ & 48 & 18 & 51 & 20 \\
$\tau=0.150$, & $8^{3}$ & 82 & 38 & 86 & 42 \\
$\kappa=0.010$, & $16^{3}$ & 79 & 42 & 82 & 43 \\
$\mu=2.0$ & $32^{3}$ & 77 & 39 & 80 & 43 \\
\hline
\end{tabular}

Table 3: Type-I closed worms: Autocorrelation times of the bulk observables at different parameters. We show the autocorrelation times $\bar{\tau}$ in units of $\tau_{0}$ and multiplied by the cost ratio $c_{s}$ as discussed in the text.

\section{$5 \quad$ Summary and discussion}

In this article we presented in detail two worm algorithms that were used in a recent QCD-inspired study of the 3-state Potts model with external field and chemical potential in three dimensions [14] and analyzed their performance. The algorithms are based on a flux representation of the model which can be obtained using high temperature expansion techniques. The nearest neighbor term leads to the conventional dimer-based closed contours, while the magnetic terms allow violation of the constraint through the insertion of monomers (similar structures will appear whenever one considers spin systems that are coupled to an external field). Furthermore, the chemical potential gives a different weight to monomers and antimonomers. Thus the model provides a rather general testbed for various algorithmic ideas based 


\begin{tabular}{lrrrrr}
\hline Parameters & $V$ & $\bar{\tau}_{\text {int }}^{U}$ & $\bar{\tau}_{\text {int }}^{C}$ & $\bar{\tau}_{\text {int }}^{P}$ & $\bar{\tau}_{\text {int }}^{\chi P}$ \\
\hline Set A: & $6^{3}$ & 14 & 6.8 & 12 & 5.1 \\
$\tau=0.100$, & $8^{3}$ & 15 & 7.2 & 13 & 5.0 \\
$\kappa=0.001$, & $16^{3}$ & 15 & 6.9 & 13 & 5.1 \\
$\mu=5.9$ & $32^{3}$ & 14 & 7.0 & 11 & 4.9 \\
\hline Set B: & $6^{3}$ & 35000 & 13000 & 1300 & 1200 \\
$\tau=0.181$, & $8^{3}$ & 31000 & 13000 & 7800 & 2700 \\
$\kappa=0.001$, & $16^{3}$ & $>25000$ & 15000 & 12000 & 3900 \\
$\mu=0.8$ & $32^{3}$ & $>19000$ & $>11000$ & $>11000$ & $>3100$ \\
\hline Set C: & $6^{3}$ & 1.2 & 0.53 & 1.1 & 0.47 \\
$\tau=0.025$, & $8^{3}$ & 1.1 & 0.50 & 1.0 & 0.46 \\
$\kappa=0.005$, & $16^{3}$ & 1.2 & 0.53 & 1.1 & 0.47 \\
$\mu=5.2$ & $32^{3}$ & 1.2 & 0.53 & 1.4 & 0.49 \\
\hline Set D: & $6^{3}$ & 2000 & 990 & 180 & 80 \\
$\tau=0.170$, & $8^{3}$ & 1400 & 740 & 210 & 100 \\
$\kappa=0.005$, & $16^{3}$ & 1200 & 580 & 230 & 100 \\
$\mu=0.2$ & $32^{3}$ & 940 & 540 & 220 & 85 \\
\hline Set E: & $6^{3}$ & 240 & 120 & 240 & 120 \\
$\tau=0.150$, & $8^{3}$ & 230 & 110 & 240 & 120 \\
$\kappa=0.010$, & $16^{3}$ & 230 & 120 & 240 & 110 \\
$\mu=2.0$ & $32^{3}$ & 230 & 110 & 240 & 110 \\
\hline
\end{tabular}

Table 4: Type-II open worms: Autocorrelation times of the bulk observables at different parameters. We show the autocorrelation times $\bar{\tau}$ in units of $\tau_{0}$ and multiplied by the cost ratio $c_{s}$ as discussed in the text. For cases marked with ">", only a lower bound could be determined.

on the worm concept [1].

We explored two types of generalized worm algorithms that differ in their treatment of monomers. The Type-I closed worms start with dimers or monomers and allow for dimer insertions or the insertion of monomers which are then followed by a random hop to another position where a second monomer is inserted. These closed worms finish when the starting position is reached again. On the other hand the Type-II open worms create chains of dimers that start and end with the insertion of monomers. It is important to note that the open worms are restricted to simulations with non-vanishing external magnetic field.

It was carefully checked that the two worm algorithms produce correct 
results, by comparison with an exact evaluation on small volumes, a crosscheck with a standard Metropolis simulation (at vanishing chemical potential), and a systematic comparison of the results from the two algorithms at various parameter values.

In order to evaluate the performance of the two algorithms we first compared various characteristic quantities, namely the starting probability $r$, the number of open segments $n_{o s}$ in Type-I worms, the average number of dimer steps $D$ of the worms, and their cost ratio $c_{s}$. We then presented the results of an analysis of the autocorrelation times, suitably scaled to reflect computational effort. The overall assessment shows that the Type-I closed worms are more flexible: They can be used also at vanishing external field and in general were found to perform better for parameter sets where monomers are scarce. Type-II open worms perform considerably better in monomer dominated regions of the parameter space. However, we expect that changing the ratio of monomer to dimer steps in Type-I closed worms would improve the latter for situations where monomers are abundant.

The authors expect that generalizations of the worm concept will see a lot of attention in the future: Matter fields (bosonic and fermionic) give rise to closed loops of flux on a lattice, and worm algorithms are a natural approach to update such a system. Of course in more realistic models the structure of the fluxes is more involved (see, e.g., the generalized effective Polyakov loop model [16]) and new strategies need to be found. We expect that the current paper is useful for such further developments. 


\section{Acknowledgments}

We thank Philippe De Forcrand, Daniel Göschl, Christian Lang, Gundolf Haase and Manfred Liebmann for fruitful discussions at various stages of this work. Y. Delgado is supported by the FWF Doktoratskolleg Hadrons in Vacuum, Nuclei and Stars (DK W1203-N08) and by the Research Executive Agency (REA) of the European Union under Grant Agreement number PITN-GA-2009-238353 (ITN STRONGnet).

\section{References}

[1] N. Prokof'ev and B. Svistunov, Phys. Rev. Lett. 87 (2001) 160601.

[2] S. Chandrasekharan and F.J. Jiang, Phys. Rev. D 68 (2003) 091501; D.H. Adams and S. Chandrasekharan, Nucl. Phys. B 662 (2003) 220.

[3] M. Fromm, J. Langelage, S. Lottini and O. Philipsen, arXiv:1111.4953 [hep-lat]; P. de Forcrand, M. Fromm, J. Langelage, K. Miura, O. Philipsen and W. Unger, arXiv:1111.4677 [heplat]; M. Fromm and P. de Forcrand, Phys. Rev. Lett. 104, 112005 (2010); PoS LATTICE2008, 191 (2008); arXiv:0912.2524] [heplat].

[4] M. Hogervorst and U. Wolff, Nucl. Phys. B 855 (2012) 885; T. Korzec, I. Vierhaus and U. Wolff, Comput. Phys. Commun. 182 (2011) 1477; P. Weisz and U. Wolff, Nucl. Phys. B 846 (2011) 316 arXiv:1012.0404 [hep-lat]].

[5] U. Wenger, Phys. Rev. D 80, 071503 (2009); U. Wolff, Nucl. Phys. B 832, 520 (2010); Nucl. Phys. B 824, 254 (2010) [Erratum-ibid. 834, 395 (2010)]; Nucl. Phys. B 814, 549 (2009); O. Bär, W. Rath and U. Wolff, Nucl. Phys. B 822, 408 (2009).

[6] L.G. Yaffe and B. Svetitsky, Phys. Rev. D 26 (1982) 963; Nucl. Phys. B 210 423. A.M. Polyakov, Phys. Lett. B 72 (1978) 477. L. Susskind, Phys. Rev. D 20 (1979) 2610.

[7] A. Patel, Nucl. Phys. B 243 (1984) 411; Phys. Lett. B 139 (1984) 394. T. DeGrand and C. DeTar, Nucl. Phys. B 225 (1983) 590. 
[8] M.G. Alford, S. Chandrasekharan, J. Cox and U.-J. Wiese, Nucl. Phys. B 602 (2001) 61.

[9] R.V. Gavai, F. Karsch and B. Petersson, Nucl. Phys. B 322 (1989) 738 .

[10] F. Karsch and S. Stickan, Phys. Lett. B 488 (2000) 319.

[11] J. Condella and C. DeTar, Phys. Rev. D 61 (2000) 074023.

[12] S. Kim, P. de Forcrand, S. Kratochvila and T. Takaishi, PoS LAT2005 (2006) 166.

[13] P. de Forcrand and O. Philipsen, Phys. Rev. Lett. 105 (2010) 152001.

[14] Y. D. Mercado, H. G. Evertz and C. Gattringer, Phys. Rev. Lett. 106 (2011) 222001; Acta Phys. Polon. Supp. 4 (2011) 703; Y. Delgado, H. G. Evertz, C. Gattringer and D. Göschl, PoS Lattice2011 arXiv:1111.0916 [hep-lat]].

[15] W. Janke and R. Villanova, Nucl. Phys. B 489 (1997) 679, and references therein.

[16] C. Gattringer, Nucl. Phys. B 850 (2011) 242. 